\title{
Strates
}

STRATES Matériaux pour la recherche en sciences sociales

14 | 2008

Espaces du quotidien

\section{Planifier le quotidien. Voisinage et unité de voisinage dans la conception des quartiers d'habitation en France (1945-1965)}

Hélène Jannière

\section{(2) OpenEdition}

Journals

Édition électronique

URL : http://journals.openedition.org/strates/6656

DOI : $10.4000 /$ strates.6656

ISSN : $1777-5442$

Éditeur

Laboratoire Ladyss

Édition imprimée

Date de publication : 1 janvier 2008

Pagination : 21-3821-38

ISBN : 0768-8067

ISSN : 0768-8067

Référence électronique

Hélène Jannière, «Planifier le quotidien. Voisinage et unité de voisinage dans la conception des quartiers d'habitation en France (1945-1965) », Strates [En ligne], 14 | 2008, mis en ligne le 04 mars 2013, consulté le 08 septembre 2020. URL : http://journals.openedition.org/strates/6656 ; DOI : https://doi.org/10.4000/strates.6656

Ce document a été généré automatiquement le 8 septembre 2020

Tous droits réservés 


\title{
Planifier le quotidien. Voisinage et unité de voisinage dans la conception des quartiers d'habitation en France (1945-1965)
}

\author{
Hélène Jannière
}

1 Dans les années 1920 à 1960, l'urbanisme planificateur s'est largement emparé de la notion de quotidien, la réduisant parfois à sa signification la plus immédiatement perceptible, celle de la journée de vingt-quatre heures, en excluant de ce fait les contenus qualitatifs ${ }^{1}$. L'étendue sémantique de la notion, ses ancrages multiples dans des pratiques (urbanisme, aménagement) et des disciplines (anthropologie, sociologie) rendent impossible une enquête sur son émergence et ses variations dans le temps ou d'un champ disciplinaire à un autre. Aussi peut-on tenter de l'aborder en se concentrant sur un objet: l'unité de voisinage, instrument de planification urbaine élaboré aux États-Unis entre les années 1910 et 1920. L'unité de voisinage est en effet à l'origine liée à la notion sociologique de voisinage, discutée dans la sociologie urbaine américaine dans les mêmes décennies. La notion de voisinage est entre autres fondée sur les rapports interpersonnels et quotidiens.

2 Apparue dans la sociologie urbaine américaine des années 1910 et 1920, la notion de voisinage a donc connu, via sa transposition en « unité de voisinage » dans l'urbanisme planificateur, une fortune importante. L'unité de voisinage s'est diffusée dans l'urbanisme américain, puis européen dès la fin des années 1930, pour connaître pendant la Reconstruction en Grande-Bretagne, Italie, Suède et Allemagne une large réception tant dans la littérature professionnelle que dans de nombreux quartiers nouveaux ${ }^{2}$. En France, elle est connue à partir de 1945; mais elle ne s'affirme réellement comme instrument de l'urbanisme planificateur qu'à la fin de la décennie suivante. Dans les années 1920, l'unité de voisinage a ainsi été conçue en rapport avec la fréquentation quotidienne - dimension qui reste très présente après 1945 - de lieux comme l'école primaire ou maternelle, ou le centre civique. Les définitions de l'unité de 
voisinage, tant britanniques que celles repérées dans les revues françaises des années 1950, insistent sur le fait que le centre communautaire (Grande-Bretagne) ou les équipements (France) de l'unité de voisinage sont de fréquentation quotidienne, alors que celles de l'échelon supérieur (quartier puis ville) sont d'usage plus rare, voir exceptionnel.

3 Cet article rappelle d'abord la signification de l'unité de voisinage pour les planificateurs lors de sa création dans les années 1920 et ses rapports avec l'idée de quotidien. Il examine ensuite le point de vue sur l'unité de voisinage des architectes urbanistes et responsables français de l'aménagement des grands ensembles, à partir du début des années 1950. Enfin, cet article aborde quelques aspects de la critique de la notion de voisinage à la fin des années 1960 par les sociologues et les observateurs des pratiques sociales et de la vie quotidienne, dans des revues destinées aux professionnels de l'aménagement ${ }^{3}$.

\section{La notion de voisinage, entre nostalgie de la communauté villageoise et « aire » de la métropole}

Dans les deux premières décennies $\mathrm{du} \mathrm{xx}^{\mathrm{e}}$ siècle, le thème du voisinage a été discuté « environ en même temps dans la planification urbaine et dans la sociologie » (Hamm, 1973, p. 11). Les deux significations de la notion de voisinage - au sens sociologique et au sens spatial - sont interdépendantes: au sens sociologique, elle se réfère aux relations qui régissent un groupement humain partageant un même territoire (area). La notion invoquée par les architectes et les urbanistes dans les nouveaux quartiers de l'après-guerre - via l'instrument unité de voisinage - a parfois été appliquée comme si, de fait, elle avait le pouvoir d'instaurer des relations de sociabilité. L'utilisation de l'unité de voisinage pour établir les dimensions d'un quartier, programmer ses équipements collectifs, prétend ainsi promouvoir automatiquement des qualités de "vie en commun». L'objectif de ce bref rappel préliminaire sur la notion ${ }^{4}$ est d'identifier les valeurs attribuées par différents auteurs, lors du transfert de cette notion de la sociologie urbaine des années 1910 et 1920 vers la planification, pour aboutir à l'unité de voisinage.

\section{Le voisinage comme ensemble de relations " primaires»}

5 Dans les premiers travaux de l'École de Chicago, le voisinage est rapporté à des formes de groupement communautaire, au sens de la communauté villageoise. Il est ainsi défini en 1909 par Charles Horton Cooley (Social Organization) ${ }^{5}$ en ligne directe de la notion de communauté élaborée à la fin du $\mathrm{xIx}^{\mathrm{e}}$ siècle en Allemagne par Ferdinand Tönnies (Tönnies, 1887) comme " groupe fondamental entre famille et groupe de jeu » (Mumford, 1968). Le voisinage se fonde sur des "relations primaires", sur la famille, étendue au village, ou à un groupement urbain de petite dimension; il se manifeste dans des relations face to face (interpersonnelles) et quotidiennes.

6 Ainsi, ce premier type de définition du voisinage se réfère à la communauté conçue comme groupe primaire (primary group), restreint et homogène, entretenant des relations intimes et constantes, cimenté par les liens de famille, incompatible enfin avec la vie urbaine. 
Les caractères qui distinguent le mieux le voisinage résident dans ses relations avec une aire locale suffisamment restreinte pour permettre un contact fréquent et intime, et l'émergence, à partir de ces relations, d'une homogénéité et d'une unité suffisantes pour permettre un groupement social primaire ou face à face, accompagné d'un fort sens de conscience de ce groupe, et capable d'influencer le comportement de ses diverses composantes (Carpenter, 1933, p. 357).

Publiée en 1933 par le sociologue américain Niles Carpenter, cette définition est emblématique de la famille de significations les plus restrictives et les plus normatives des relations de voisinage, où celui-ci est conçu comme une entité antinomique à la grande ville. Elle distingue en outre le generic neighborhood (véritable voisinage) du pseudo-neighborhood, c'est-à-dire des aires résidentielles existant dans la grande ville et non des aires de voisinage ${ }^{6}$. Enfin, dans cette conception, le voisinage ne peut exister que dans des conditions de mobilité réduite : la vie dans les métropoles détruit les relations $\mathrm{du}$ groupe premier; lorsque la mobilité physique et sociale des membres de la communauté s'accroît, l'entité " voisinage » tend à se dissoudre. Ce type de définition se réfère à des formes d'organisation traditionnelle, où la communauté villageoise est érigée en modèle idéal et indépassable du voisinage.

\section{Voisinage et comportements citadins}

8 Un second type de définition se rattache davantage aux réflexions des mouvements réformistes américains qui tentent de répondre aux problèmes sociaux engendrés par la croissance incontrôlée des villes. Ainsi, l'objectif du Community Center Movement est de répartir dans les aires naturelles de voisinage existant à l'état spontané au sein de la grande ville ${ }^{7}$, des équipements collectifs censés créer des lieux d'intégration sociale en attribuant à chaque petite communauté « un endroit destiné aux rencontres locales en commun, qui puisse offrir un forum pour les discussions et servir de base aux activités de la communauté, qui, sans cela, ne trouve pas de lieu d'accueil» (Mumford, 1968, p. 62). Pour E. W. Burgess, qui prend en compte la mobilité sociale et physique inhérente aux modes de vie citadins en un ensemble de relations dynamiques, un ensemble de forces en interaction, il existe des voisinages à l'état «naturel» dans la grande ville :

In the study of the growth of the city it is found that the life of any neighborhood is determined, in the long run, not altogether by the forces within itself, but even more by the total course of city life. To think of the neighborhood or the community in isolation from the city is to disregard the biggest fact about the neighborhood (1925, p. 148).

9 La notion de voisinage envisage alors des relations secondaires liées aux comportements citadins. Vue dans la conception restrictive de la notion de voisinage comme un facteur de désorganisation sociale et une menace pour la communauté, la mobilité est considérée par plusieurs auteurs de l'École de Chicago (en particulier Robert E. Park $^{8}$ ) comme une caractéristique positive des aires de la ville moderne.

Pour Lewis Mumford enfin, l'un des partisans de la théorie de l'unité de voisinage, le voisinage ne réside pas dans une origine commune ou des buts communs de ses habitants mais dans la proximité spatiale, celle-ci se manifestant dans la « conscience de l'autre ", la vue, la communication, l'association et la coopération en cas de crise (Mumford, 1968). 


\section{Du voisinage à l'unité de voisinage}

11 L'introduction de la notion de voisinage dans le domaine de la planification urbaine s'effectue au début des années 1920, aux États-Unis.

12 Attribuée par la plupart des commentateurs à l'un des leaders de la Regional Planning Association, Clarence Arthur Perry (1872-1944)9 , la paternité de l'unité de voisinage n'est toujours pas tranchée aujourd'hui. Pour plusieurs analystes, C. A. Perry n'« invente pas le concept de neighborhood unit » mais retraduit et élabore de manière articulée à un principe de planification une idée présente dans la théorie de la citéjardin d'Ebezener Howard en 1898 (Dahir, 1950, p. 212). D'autres commentateurs attribuent à d'autres " précurseurs » la mise au point de cet instrument (Klages, 1968). Une analyse récente (Johnson, 2002) accorde l'invention de l'unité de voisinage à un architecte, William E. Drummond, lors d'un concours organisé en 1912-1913 pour la ville de Chicago par le City Club, visant à établir un contre-projet au plan de Daniel

H. Burnham et Edward H. Bennett. D'après cette interprétation, W. E. Drummond aurait défini le terme " unité de voisinage », donnant ainsi forme au voisinage alors étudié par les sociologues de l'École de Chicago. L'auteur Donald L. Johnson affirme que l'intérêt pour la notion de voisinage était très répandu aux États-Unis de 1898 à 1916, et que C. A. Perry se l'est en suite appropriée et l'a diffusée.

14 Selon L. Mumford à l'inverse, C. A. Perry a le premier tenté de donner une cohérence à une communauté en pourvoyant de "centres communautaires» promus par le Community Center Movement, non plus les communautés ou voisinages spontanés existant à l'état latent dans les grandes villes, mais à une entité recréée, structurée suivant les définitions du voisinage récemment élaborées par l'École de Chicago ${ }^{10}$.

[C. A. Perry] redonnait ainsi au voisinage les fonctions qui avaient commencé à tomber en désuétude, ou qui, depuis le déclin de la ville médiévale, étaient devenues, à tort, centralisées. Ce pas le mena du voisinage à l'unité de voisinage : d'une pure cohabitation, à la création d'une nouvelle forme et de nouvelles institutions destinées à une communauté urbaine moderne (Mumford, 1968, p. 63).

$\mathrm{Au}$ voisinage, naturel et spontané caractéristique des communautés villageoises et de la ville médiévale, ou latent au sein de la ville moderne, C. A. Perry décide de substituer un voisinage recréé artificiellement par une organisation spatiale répondant à des critères précis.

Nous n'avons pas, dans les limites de cet article, les moyens de trancher sur cette attribution. L'importance de C. A. Perry tient notamment à la formalisation du modèle et à sa diffusion dans les milieux réformateurs américains. Exposée en 1923 pour la Russell Sage Foundation (Dahir, 1950, p. 212), puis détaillée en 1929 dans le volume 7 de Regional Survey of New York and its Environs (Perry, 1929), l'unité décrite par C. A. Perry et c'est ce modèle qui a ensuite été cité et diffusé - dérive des «besoins de la vie familiale ».

17 C. A. Perry insiste sur les rythmes différents de ces besoins, et notamment sur leur quotidienneté :

Pour investir en bourse, aller à l'opéra, visiter un musée, qui sait pour acheter un piano, on doit aller " au centre ». Mais il y a d'autres services, fonctions ou aspects strictement locaux et typiques d'une communauté résidentielle bien organisée. Ils peuvent être classés en 4 parties : 1) l'école élémentaire, 2) des petits parcs et aires de jeux, 3) des commerces locaux, 4) des résidences (Perry, 1929). 
Le centre de l'unité de voisinage est constitué du common, "espace vert communautaire» (Perry, 1929), sur lequel donnent un édifice scolaire et deux constructions latérales, par exemple une bibliothèque publique et un lieu de culte (figure 1).

Figure 1. Clarence Arthur Perry. Schéma de I'Unité de voisinage, 1923.

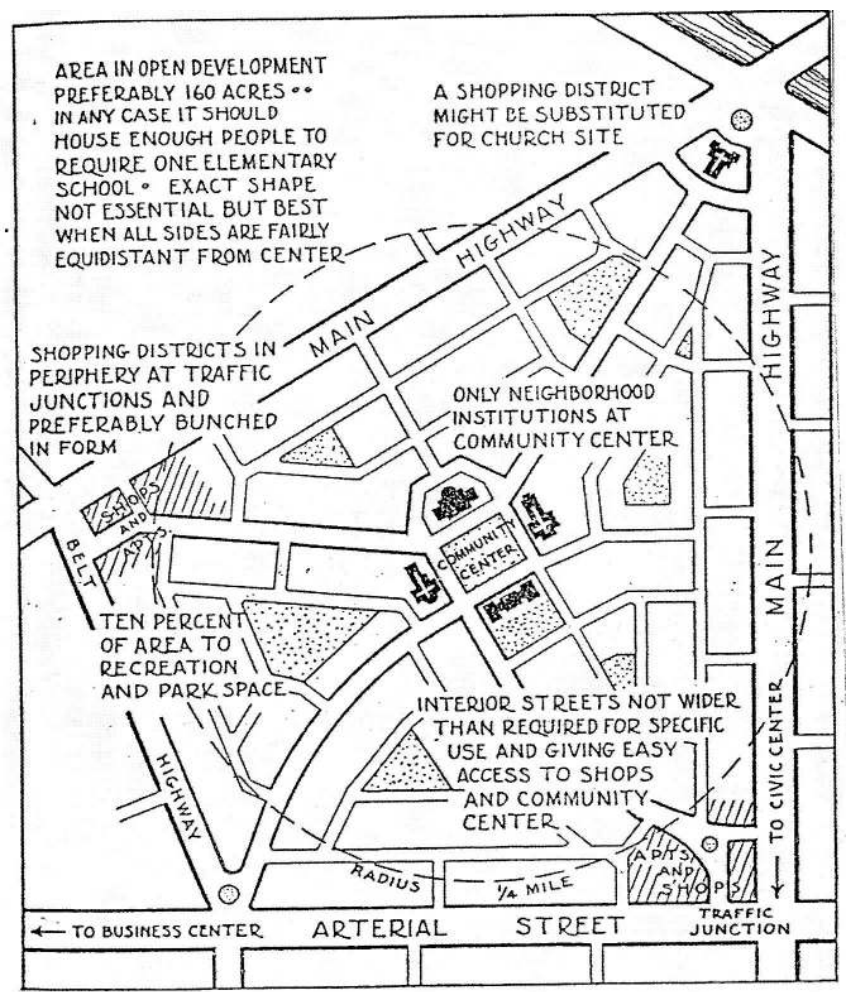

Publié dans «The Neighbourhood Unit », Regional Survey of New York and its Environs, New York, vol. VII, 1929.

Le premier principe édicté par C. A. Perry est le nombre d'habitants, limité par les capacités d'un équipement scolaire : 5000 à 6000 habitants, ce qui correspond à une école de 1200 élèves $^{11}$. De faible densité, l'unité de voisinage ne peut ainsi pas s'étendre mais sa croissance doit être absorbée par une autre unité. Deuxième principe, l'unité de voisinage, structurée par l'étude des déplacements de l'enfant, s'inscrit dans un cercle autour du centre communautaire, c'est-à-dire l'école, distant de tout point d'un demimile (environ 800 mètres). Elle doit pouvoir être traversée à pied.

Rejetées à l'extérieur, les circulations de transit forment les limites de l'unité et accueillent les commerces de fréquentation quotidienne, accessibles à pied (Perry, 1926). Seules les voies de desserte purement locale, « conçues pour mener au centre communautaire, ajoutant au sens d'unité physique» (Dahir, 1950, p. 214) la traversent. C. A. Perry insiste enfin sur le pourcentage (minimum $10 \%$ ) d'espaces libres: espaces de proximité, d'usage quotidien, ils sont destinés une fois encore à l'enfant (open space, recreation space, park space). De surface plus restreinte mais plus nombreux, ils se substituent aux parcs traditionnels dont l'usage ne peut être quotidien.

21 Après le quartier de Radburn (New York, 1928-1933), conçu par Clarence S. Stein, Henry Wright, Frederick L. Ackermann, architectes et urbanistes proches de l'École de Chicago, le principe de l'unité de voisinage a été appliqué dans les Greenbelt Towns de 
la Tenessee Valley Authority. L'unité de voisinage a trouvé dans ces villes nouvelles un terrain d'application particulièrement propice, la politique urbanistique et d'aménagement du New Deal voulant associer modèle social à modèle spatial ${ }^{12}$.

\section{La diffusion de l'unité de voisinage en France}

Connue en France depuis 1945, l'unité de voisinage est diffusée sur une grande échelle surtout à partir de 1958, lors de la promulgation du décret sur les ZUP ${ }^{13}$. À ce stade de la recherche, les éléments présentés sur la réception de l'unité de voisinage par les architectes et les urbanistes français sont principalement issus de l'analyse du périodique Urbanisme, l'un des supports privilégiés des professionnels de l'aménagement après la Seconde Guerre mondiale, qui publie plus de grands ensembles que les revues d'architecture. Urbanisme répercute en outre le discours officiel et les directives du ministère de la Reconstruction et de l'Urbanisme, devenu en 1958 ministère de la Construction. Les ouvrages d'urbanisme de la période 1945 à 1960 sont assez nombreux à employer l'expression "unité de voisinage ", souvent comme synonyme de grand ensemble ou d'unité résidentielle, mais finalement peu nombreux à commenter cet instrument de planification. De cette lecture, se dégagent plusieurs constats.

Figure 2. L'Unité de voisinage et son application dans la ville nouvelle de Harlow (Grande-Bretagne).
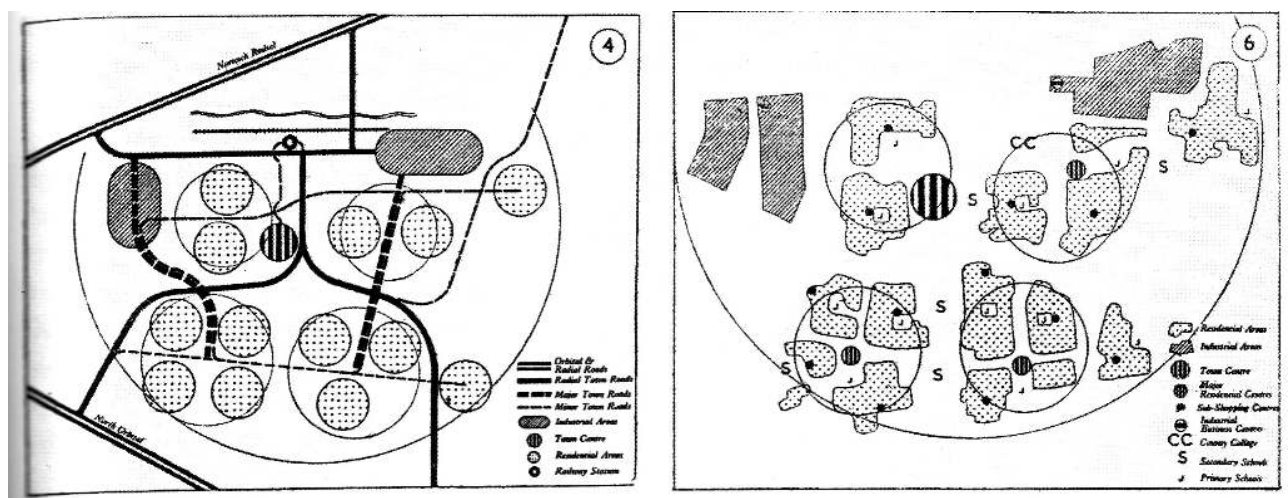

À gauche, la structure de la ville et les voies de circulation ;à droite,groupement des unités de voisinage et leur structuration autour des écoles.Schémas publiés dans Urbanisme n 1-2, 1950, p. 47. (c) Urbanisme

La réception de l'unité de voisinage est plus tardive en France que dans d'autres pays européens : aucun quartier construit avant la Seconde Guerre mondiale, ni même avant 1950, ne se nomme « unité de voisinage ». En Angleterre, s'inscrivant en continuité du mouvement pour les cités-jardins, la théorie se diffuse dans les milieux aménageurs. L'instrument neighborhood unit est intégré en 1937 dans les prescriptions du Town Planning Act et appliqué dès la première phase des villes nouvelles, de 1946 à 1951 (figure 2).

En Italie, dans un contexte de découverte de la sociologie urbaine américaine, en particulier de L. Mumford et de l'École de Chicago ${ }^{14}$, l'architecte et critique Bruno Zevi publie dans sa revue Metron (1945-1954) la théorie de l'unité de voisinage en 1946 (Calandra, 1946). Parallèlement, de nombreux quartiers dans les périphéries romaine ou turinoise (ex. La Falchera près de Turin, figure 3) obéissent à ses prescriptions. Dans 
ces ensembles, à la structuration du quartier par l'unité de voisinage, s'associe sur le plan architectural une rupture avec le langage rationaliste de l'avant-guerre au profit d'un langage vernaculaire considéré comme expression spontanée du " peuple ». Dans cette phase de reconstruction sociale et politique de l'Italie, cette recherche d'une forme d'« authenticité " s'appuie sur le repli sur des valeurs de sociabilité de petites communautés, avec pour modèle les villages du Sud ${ }^{15}$. Enfin en Allemagne ${ }^{16}$, tant la construction de nouveaux quartiers que les propositions de modèles théoriques de "ville organique ", dédensifiée, organisée en petites communautés (Reichow, 1948) ${ }^{17}$ remettent à l'honneur la notion de voisinage au lendemain de 1945. Une telle redécouverte (renforcée par la réception du Plan de Londres de 1944) prétend porter ainsi, comme en Italie, des valeurs « démocratiques » dans la Reconstruction, mais elle renoue ici avec des modèles élaborés pendant les années 1940. Dans ces trois exemples cités trop brièvement, il faut noter l'association de l'unité de voisinage avec la volonté de retour à des petites communautés d'habitants, et la référence directe à la théorie élaborée par C. A. Perry. Si dès 1945, l'unité de voisinage est connue en France par le biais de la publication des villes nouvelles britanniques, les premiers articles d' Urbanisme(Suquet-Bonneaud, 1950) (figure 4) la mentionnant ne font pas référence aux conceptions de C. A. Perry. L'influence anglo-américaine semble donc d'autant plus limitée chez les architectes et urbanistes français que dès le début des années 1950, la théorie de l'unité de voisinage entre dans la conception des grands ensembles, s'associant ainsi à une vision fonctionnaliste de la conception des quartiers d'habitation. À l'inverse de la plupart des quartiers planifiés suivant cette théorie en Italie et en Grande-Bretagne - vernaculaires ou pittoresques, proches de l'esthétique de la cité-jardin -, l'architecture des quartiers français est en outre le plus souvent moderniste. Une seule exception, qui se rapproche davantage des conceptions angloaméricaines de l'unité de voisinage, la théorie des échelons de Gaston Bardet. 
Figure 3. Giovanni Astengo, Alessandro Molli-Boffa, Mario Passati, Nello Renacco et Aldo Rizzotti (urbaniste). Quartier INA-Casa « La Falchera»,Turin,1 1950-51.

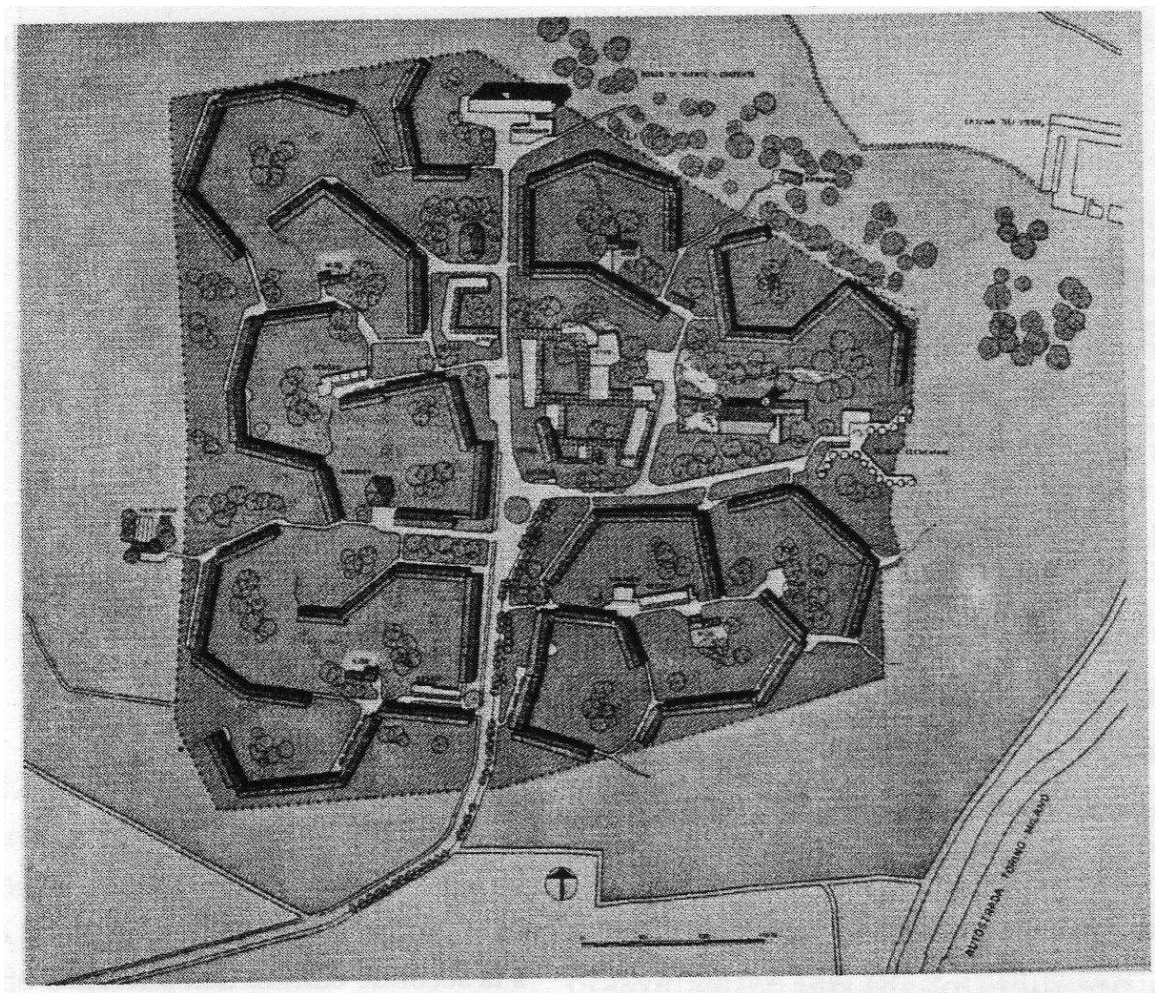

(C) Archivio Agenzia Territoriale per la Casa, Turin. DR

\section{Les « échelons communautaires » de Gaston Bardet}

Dans les années 1930, G. Bardet a développé l'idée de "topographie sociale », méthode censée mettre visuellement en évidence la structure sociale de la ville et ses groupements humains. La première édition de Problèmes d'urbanisme (1941) évoque la notion d'unité résidentielle (à partir de l'exemple de Radburn et des Höfe viennois). Dans Pierre sur pierre (1946, recueil d'écrits de 1934 à 1943), et dans Le Nouvel urbanisme (1946), G. Bardet développe la théorie des «échelons communautaires » élaborée en 1943, fondée sur une représentation organiciste de la ville redevable à la littérature urbanistique anglo-américaine, dont il est l'un des rares passeurs en France. Trois échelons (patriarcal, domestique, paroissial) composent en s'emboîtant une communauté organique (Bardet, 1948). À l'échelon patriarcal est associée la société familiale (échelon du "groupe familial de voisinage », défini par G. Bardet comme un " coude à coude de 15 à 20 ménages »); à l'échelon domestique, la société économique (échelon délimité comme " périmètre de cheminement de la ménagère ", composé d'un fragment de petit quartier); à l'échelon paroissial, la société politique (échelon qui représente "l'évocation du rôle communautaire de la paroisse»). Apparemment passéiste, cette conception n'est pas si éloignée des conceptions restrictives du voisinage qui déterminent le fonctionnement de l'unité de C. A. Perry. G. Bardet précise que le dernier échelon dépasse l'échelle des activités quotidiennes et que "les AngloSaxons lui donnent le nom d'“unité de voisinage" » (1957), introduisant en ce sens une variation par rapport à la définition américaine de l'unité. Pour G. Bardet, un urbanisme fondé non sur le plan-masse dessiné a priori, régi par la géométrie, mais sur 
les échelons permet d'« utiliser l'étroite communauté de destin que crée la proximité dans les petites sociétés closes, sans oublier la nécessité des aspirations vers les grandes sociétés ouvertes : telle doit être la doctrine des urbanistes de la seconde moitié du $\mathrm{xx}^{\mathrm{e}}$ siècle » $(1948$, p. 249).

Figure 4. Antoinette Suquet-Bonneaud, « L'aménagement des quartiers de résidence en Angleterre», Urbanisme, $n^{\circ} 1-2,1950$, p. 35 et p. 48.

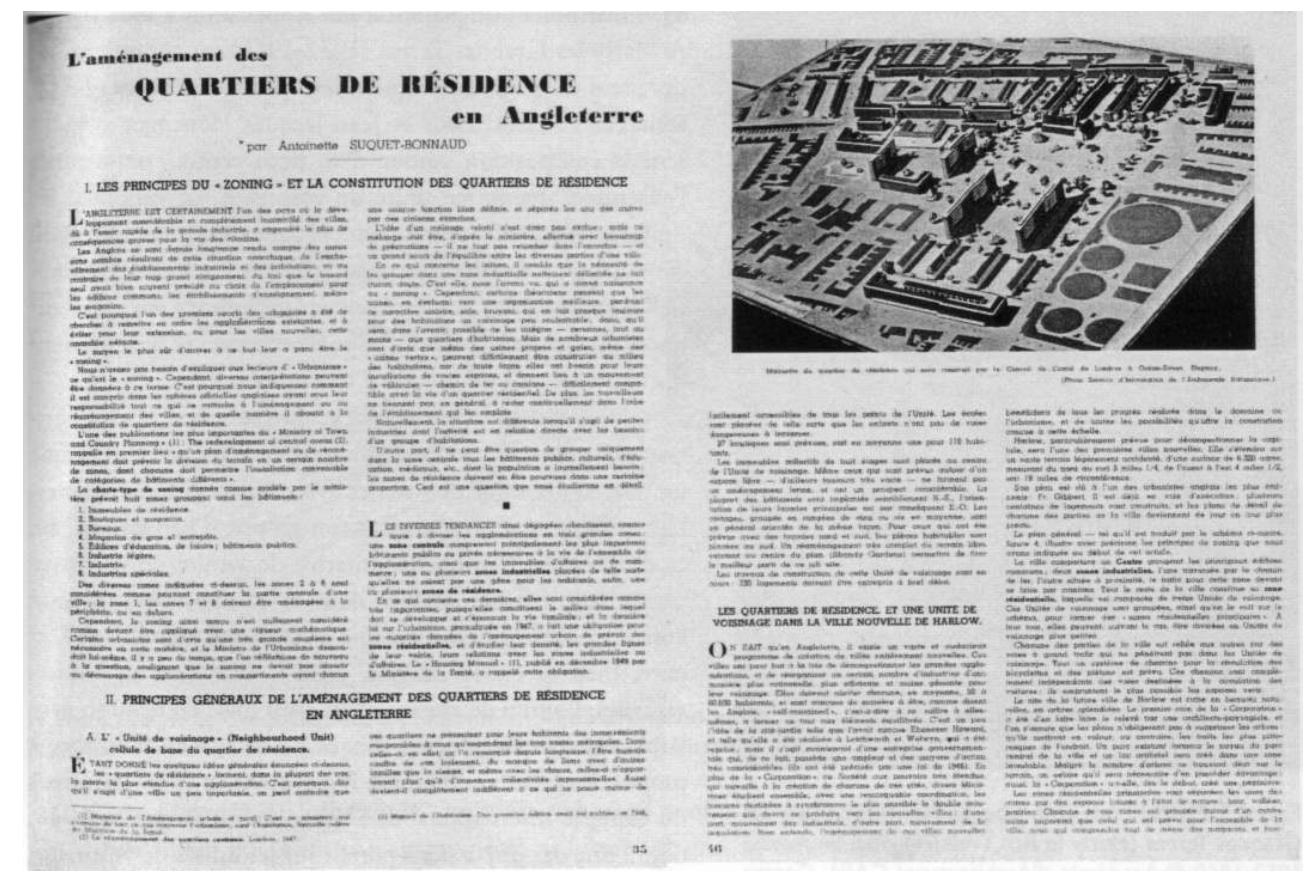

(c) Urbanisme

En 1950, G. Bardet continue de proposer une méthode de conception des grands ensembles (leur « organisation polyphonique ») (Bardet, 1950) à partir de la théorie des échelons qu'il publie en 1957 dans Urbanisme, prenant le contre-pied des doctrines fonctionnalistes. Le terme d'échelon est repris par Paul-Henry Chombart de Lauwe dans plusieurs articles d'Urbanisme en 1959. Ce terme confère un contenu social à l'unité de voisinage, alors que celle-ci est alors en train de devenir une subdivision technocratique entre unité résidentielle et unité de quartier.

\section{Unité de voisinage et traitement architectural}

Entre 1950 et 1958, le terme d'« unité de voisinage » est employé assez sporadiquement tant par les pouvoirs publics que les concepteurs. Flottant, il est alors synonyme d'ensemble ou de quartier: il désigne ainsi les ensembles de Bron-Parilly ${ }^{18}$, de Vernouillet ${ }^{19}$, de Bordeaux-la-Bénauge par exemple sans que leurs concepteurs se réfèrent explicitement à cet instrument de planification. Appellation «abusive ${ }^{20}$ ? À Marly-les-Grandes-Terres (1952-1960) au contraire, l'un des premiers ensembles dénommés " unité de voisinage », les architectes Marcel Lods et Jean-Jacques Honegger structurent la composition autour d'un petit centre commercial ; l'ensemble, dénommé unité de voisinage, est subdivisé en unités résidentielles regroupant chacune trois immeubles peu élevés autour d'un espace vert. (figure 5) Les unités résidentielles sont 
lisibles tant en plan qu'en volume, ou elles forment des espaces semi-clos, pouvant renforcer le sentiment d'appartenance à un groupe d'habitations.

Figure 5. Marcel Lods, Jean-Jacques Honegger, Marly les Grandes Terres (Marly le Roi,Yvelines), plan de masse, 1952-1960.

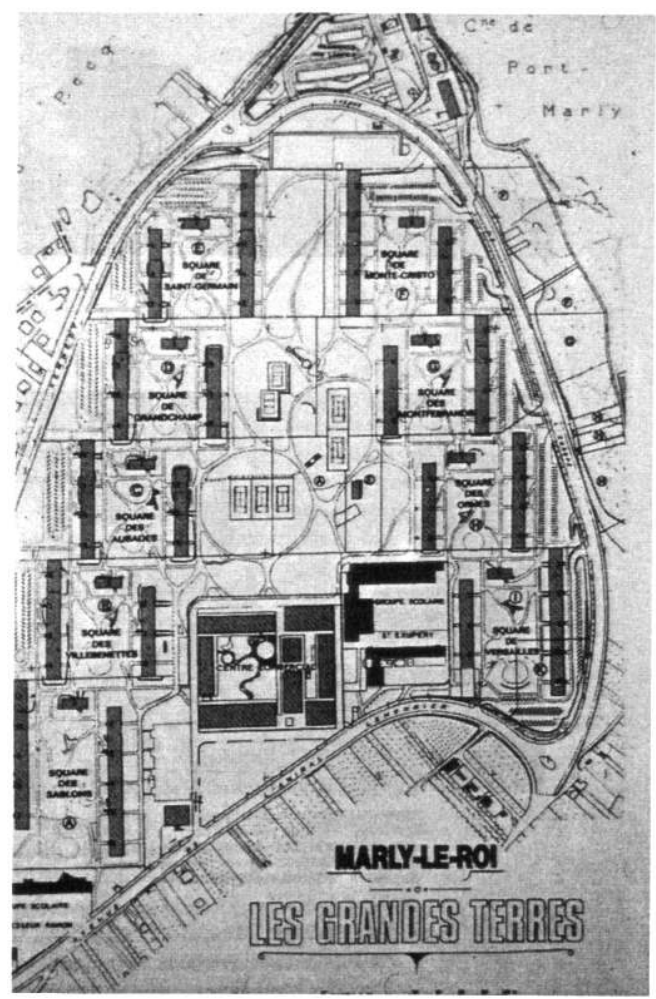

(c) Académie d'Architecture/CAPA, Centre des archives de l'architecture du XXe siècle, fonds Marcel Lods, 323AA, DR

De 1950 au début à la décennie suivante, la composition des volumes architecturaux est en effet perçue comme un moyen plastique de susciter le sentiment d'appartenance, l'identification de l'habitant à son groupe d'habitations. En ce sens, la subdivision quantitative de l'unité de voisinage trouve une correspondance dans la composition, divisant l'open space du grand ensemble en des sous-unités dont l'espace, bien qu'encore ouvert, offre la sensation de limites visuelles. L'unité de voisinage est ainsi mise en relation avec la plastique architecturale, thème qui monte en puissance à propos des grands ensembles. Rapportant en 1950 les débats du Congrès international de l'Urbanisme à Zurich de 1948, dont l'une des discussions portait sur les unités de voisinage, Urbanisme en montre la double acception, tantôt découpage quantitatif ( "module pour bâtir une ville et lui faire remplir ses fonctions »), tantôt porteuse de cohésion sociale ( Il y a aussi ceux [les urbanistes] qui mettent l'accent sur les affinités qui se développent parmi les habitants et qui croient que ces unités correspondent à des groupes sociaux existants ou nouveaux $\left.{ }^{21} »\right)$. L'unité de voisinage ne se limite pas ici à une échelle intermédiaire fonctionnelle qui structure mécaniquement l'ensemble d'habitation. À unité sociale et fonctionnelle, s'ajoute la nécessité d'« unité architecturale » :

Si nous désirons susciter la naissance de nouveaux groupes sociaux, les limites de l'unité résidentielle doivent être accessibles à l'œil. C'est aussi important que la création d'un centre de vie communautaire. Ce centre, et les bâtiments 
correspondants dans les unités plus larges, doit être traité architecturalement avec des formes et des couleurs plus intenses et plus expressives que celle des habitations ${ }^{22}$ (figure 6).

Figure 6. Claude Le Cœur, Esquisse d'Unité résidentielle à Cronenbourg (publiée dans Urbanisme, $\left.\mathrm{n}^{\text {os }} 1-2,1950, \mathrm{p} .29\right)$ pour illustrer les débats sur l'Unité de voisinage au Congrès international de l'Urbanisme à Zurich de 1948.

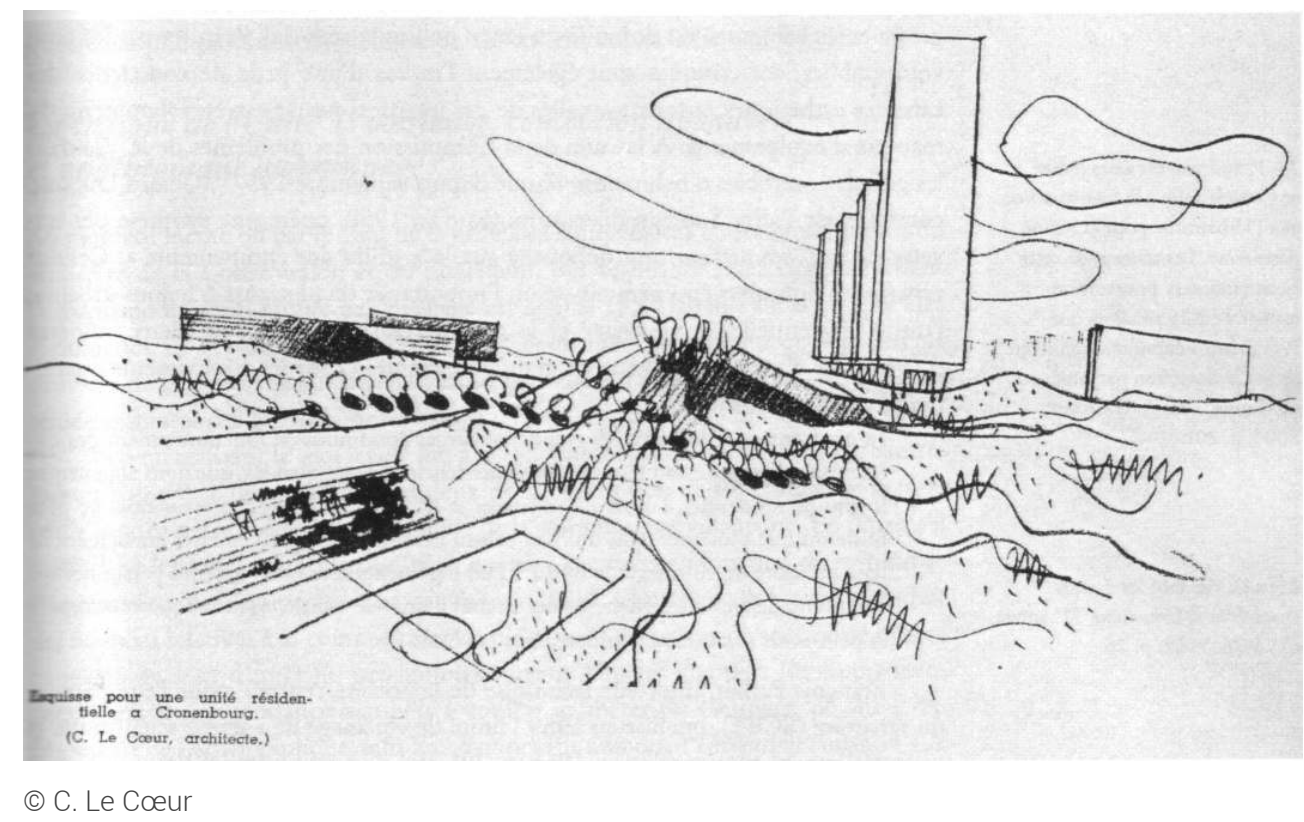

Cette exigence de lisibilité de l'unité de voisinage rejoint les conceptions de C. A. Perry, bien que celui-ci n'ait privilégié ni forme urbaine, ni caractères architecturaux : pour le planificateur américain, l'unité doit "permettre au public de voir une communauté locale en tant que telle et de la reconnaître comme une entité de quartier » (1929). Son identification s'appuie sur la visualisation de ses frontières (route, canal, élément naturel) et sur l'expression architecturale conférée au centre communautaire qui « [...] doit devenir un point de focalisation, autant physiquement que psychiquement, pour la vie du voisinage ${ }^{23} »$. Deux éléments, limites visuelles et marquage de l'équipement, que l'on retrouve dans la littérature concernant les grands ensembles. Dans plusieurs écrits du début des années 1960, l'architecte français Xavier Arsène-Henry évoque lui aussi la lisibilité des échelons urbains par le traitement architectural monumental des équipements - «note de variété " (Arsène-Henry, 1962, p. 22) semée entre les habitations - et par la différenciation des voieries, leur hiérarchie, qui doivent « limiter l'horizon » de l'utilisateur et lui faire «prendre conscience de l'échelon dans lequel ils évoluent » (p. 23).

À la fin des années 1950 et lors de l'instauration de la Ve République, la réussite des grands ensembles, critiqués depuis 1955 par les associations familiales, la presse et les habitants, est devenue un enjeu politique et social. Reprises par les pouvoirs publics, ces critiques sont également l'indice d'une prise de conscience des carences esthétiques et fonctionnelles de ces quartiers nouveaux et notamment, du manque d'équipements. À la suite de la Commission des problèmes de la Vie dans les grands ensembles d'habitation, réunie depuis septembre $1957^{24}$, Gérard Dupont, conseiller de Pierre Sudreau devenu ministre en 1958, opère une synthèse des travaux de la Commission, qui débouche sur la «grille des équipements». Celle-ci répartit les différents 
équipements selon l'importance de plusieurs échelons urbains : l'unité résidentielle, le voisinage et le quartier. En 1959 et 1962, deux numéros d'Urbanisme respectivement sur les grands ensembles et sur les équipements quantifient l'unité de voisinage :

La confrontation des données recueillies sur les conditions de fonctionnement des différents équipements fait ressortir plusieurs échelons remarquables, que nous appellerons le groupe résidentiel, l'unité de voisinage et le quartier. Ce groupe résidentiel ne peut toutefois que s'intégrer dans un tissu urbain existant. Il apparait en effet un élément de base ou unité de voisinage de 800 à 1200 logements soit 3000 à 4500 personnes qui comprend déjà tous les éléments nécessaires à une vie sociale, économique et culturelle et peut avoir son caractère propre dans la ville ${ }^{25}$.

31 François Parfait, directeur technique de la Société centrale pour l'équipement du territoire (SCET), qualifie en 1959 l'unité de voisinage de "terme scientifique et universellement admis » (Parfait, 1959, p. 20), afin de préciser les échelles respectives et du grand ensemble et de l'unité de voisinage, les hiérarchies et le caractère de cohésion d'un ensemble d'habitations, ce qui différencie l'unité de voisinage d'une quelconque opération de logement. Or, ni la définition et ni l'échelle de l'unité de voisinage ne font l'unanimité: P.-H. Chombart de Lauwe conteste le caractère trop vaste de l'unité utilisée par les pouvoirs publics et les urbanistes, dimension dans laquelle se dilue la cohésion sociale attendue.

C'est ainsi que l'unité de voisinage tend à devenir un instrument de découpage quantitatif du grand ensemble. Elle est associée à une échelle d'équipements et à des fonctions de rythme quotidien (concrétisées par des services tels que la "supérette", l'école maternelle ou primaire). Elle apparaît comme un schéma rationnel et technique d'organisation des habitations, et comme une échelle minimum d'opération qui, à partir de 1951 (date du concours du quartier Rotterdam à Strasbourg) permet l'organisation du chantier industrialisé (Dieudonné, 1996). Cette hiérarchie reprend en partie le système pyramidal des échelons de G. Bardet, mais en le vidant de ses contenus de sociabilité et de sa référence à une communauté cohérente et soudée. Il est paradoxal que dans son utilisation, l'invocation des potentialités de cohésion communautaire, d'échanges, de rencontres qui faisaient de l'unité de voisinage l'instrument d'une cohésion sociale espérée disparaisse, alors qu'en France à la fin des années 1950, l'unité de voisinage a été convoquée dans une politique d'amélioration de ces ensembles déjà tant décriés.

\section{La critique de l'unité de voisinage, conception appauvrie et anachronique du voisinage?}

33 Au moment même où par le biais de la grille des équipements et des prescriptions du ministère de la Construction et du Logement, elle se diffuse assez largement dans l'urbanisme français, l'unité de voisinage est depuis 1958 la cible de la critique des sociologues, en France comme à l'étranger. Ces critiques portent sur deux points : la pauvreté de la notion et son anachronisme, le doute sur son efficacité, lorsqu'elle est censée instaurer des relations de voisinage.

Bien qu'utilisant le mot «échelon », P.-H. Chombart de Lauwe puis l'urbaniste Robert Auzelle se démarquent tous deux de la conception de G. Bardet, en grande partie fondée sur le modèle anachronique de la communauté villageoise. En outre, s'il hiérarchise les échelons (voisinage, petit quartier, quartier) en fonction du rythme de 
fréquentation des équipements et commerces, P.-H. Chombart de Lauwe introduit des nuances relatives à la composition sociologique. Les formes de sociabilité propre au voisinage sont d'après lui perceptibles surtout dans les quartiers les moins favorisés. Il existe une tendance naturelle à vouloir se libérer du voisinage, ce que P.-H. Chombart de Lauwe explique dans les colonnes du numéro d'Urbanisme consacré aux équipements. Il reprend en 1963 dans Des hommes et des villes :

Il semble que la tendance à reconstituer des "villages " s'oppose au besoin de se libérer des contraintes du voisinage, que la recherche de la stabilité s'oppose aux habitudes de mouvement perpétuel de certains citadins. [...] La vie quotidienne du petit quartier prolétarien de zone stable donne bien en effet par rapport à un quartier bourgeois aisé l'impression d'un autre monde. C'est une petite unité économique de consommation et un groupe de voisinage dont nous ne trouvons pas d'équivalent de l'autre côté au même échelon (Chombart de Lauwe, 1963, p. 34-36).

Cet anachronisme avait été précédemment relevé par la sociologie urbaine américaine et allemande. Le recours à l'unité de voisinage a été massif dans la reconstruction allemande, ce qui explique l'abondance des critiques des résultats obtenus dans ce pays, où des ouvrages entiers sont consacrés au voisinage et à l'unité de voisinage (Hamm, 1973; Klages, 1968; Pfeil, 1964 et 1972). Ils relèvent d'abord l'anachronisme des définitions les plus restrictives du voisinage, lors de son application après guerre aux quartiers de reconstruction. Mais surtout, selon ces auteurs, il n'y pas de traces de l'espace social de la communauté dans le schéma de C. A. Perry, établi à partir de considérations fonctionnelles liées à la vie quotidienne, à la circulation quotidienne piétonne et automobile à partir de l'école, élément fédérateur. Les fonctions de la vie quotidienne sont déduites de l'observation des besoins de la cellule familiale, où l'enfant tient une place prépondérante. La plupart des distances sont réglées en fonction de ses déplacements quotidiens, de la maison à l'espace libre le plus proche (playground), de la maison à l'école maternelle puis primaire (Mann, 1965). C. A. Perry fait en outre l'hypothèse de la création de relations immédiates et spontanées du fait d'appartenance au même voisinage (relations primaires, face-to-face contact).

Dès la fin des années 1950, plusieurs de ces sociologues allemands stigmatisent comme très faibles les représentations sociales du voisinage chez $\mathrm{C}$. A. Perry :

Si le quartier signifiait pour les sociologues de l'École de Chicago en premier lieu l'espace social du voisinage, la zone d'habitation de C. A. Perry est un espace, qui concentre des conveniences and amenities répondant à des besoins individuels. Du « voisinage » au sens de relations sociales réciproques liées à l'échelle régionale, il ne reste ici aucune trace (Klages, 1968, p. 21).

Cette critique peut-être trop radicale à l'égard du schéma de $C$. A. Perry pourrait néanmoins être appliquée au cas de la France : à l'appauvrissement des conceptions du voisinage lors de sa transformation en instrument urbanistique, s'ajoute ici l'altération de l'unité de voisinage elle-même, par rapport au modèle anglo-américain, plus souple et considéré comme instrument de conception architecturale et paysagère.

En France, la critique de l'unité de voisinage est l'un des volets, mais loin d'être le plus diffusé, de la critique des grands ensembles. Le premier type de critique concerne la pauvreté de la conception de ces relations sociales. Henri Lefebvre fustige à juste titre un usage instrumental de l'unité de voisinage - et au-delà, de la sociologie dans son ensemble - par les responsables français de l'aménagement. Le deuxième trait est le fait, déjà relevé dans la décennie précédente tant par L. Mumford que par les sociologues urbains allemands, que le recours à l'unité de voisinage dans les quartiers 
neufs semblerait pour les concepteurs des années 1945 à 1960 induire de fait des qualités de sociabilité pour ces quartiers, des relations de voisinage, des modes d'interaction sociale, peu ou faiblement évoquées dans les textes des urbanistes. Mais la réalité de ce transfert est dès les années 1960 fortement critiquée par la sociologie urbaine, qui souligne les différences entre le voisinage au sens social et au sens spatial (neighborhood et neighboring, deux niveaux que la langue anglaise distingue, différenciation reprise par la critique allemande ${ }^{26}$ ). H. Lefebvre témoigne de cette même préoccupation, lorsqu'il déclare à propos de Mourenx :

À juste titre, on se soucie d'incarner dans le temps et dans l'espace (historiques) la collectivité nouvelle, de transférer sur elle et en elle la sociabilité spontanée déjà en acte. Est-on donc sûr que la sociabilité spontanée se conserve, se retrouve, s'investisse dans l'agglomération nouvelle? Il n'est pas certain que des relations de voisinage [...] s'établissent entre voisins rapprochés par le hasard, membres accidentels d'une unité d'habitation, sans arrière-plan historique intervenant dans les vécus. L'unité créée devient l'appendice artificiel et mécanique d'une collectivité organique [...] (1960, p. 187-188).

\section{Conclusion}

À la fin des années 1950, s'était forgée l'idée du grand ensemble comme «laboratoire social », dans lequel se seraient élaborées « les structures sociales de demain ", c'est-àdire aurait dû naître une nouvelle sociabilité grâce au mélange des classes sociales dans un environnement neuf. Dans cette optique, l'unité de voisinage pouvait offrir une forme de cohésion à ces quartiers. Des titres d'articles comme «Le grand ensemble, facteur de progrès social " (Dupont, 1959) révélaient cet optimisme, en même temps qu'était à l'époque envisagée l'« amélioration » des grands ensembles, concrétisée dans la législation par le décret sur les ZUP et dans les prescriptions par la grille des équipements. En associant en 1958 l'unité de voisinage à la grille des équipements, instrument technocratique censé pallier les défauts des grands ensembles, en invoquant la notion de voisinage, les responsables français de l'urbanisme ont conféré indirectement à l'unité de voisinage dans les années 1950 et au début des années 1960 en France le pouvoir de remédier à plusieurs carences des grands ensembles : l'absence d'équipements, le manque d'autonomie des quartiers et les difficiles relations sociales dans ces quartiers neufs (les problèmes des femmes, «transplantées » dans le grand ensemble, et ceux des jeunes, sont le plus souvent cités). Or, cette conception "quantitative » de l'unité de voisinage, jouant un rôle dans le dimensionnement des différents échelons et des équipements nécessaires, s'ajoute à une compréhension restrictive et anachronique des relations de voisinage incarnées par l'unité dès sa conception par C. A. Perry. Encore à la fin des années 1950, l'unité de voisinage était en effet, sporadiquement il est vrai, référée dans la revue Urbanisme $e^{27}$ aux premières définitions du voisinage, se référant au "groupe premier ", à la communauté familiale ou villageoise qu'il s'agissait d'artificiellement restaurer dans les ensembles d'habitations.

Rien d'étonnant à ce que, à la fin des années 1960, des conceptions à la fois aussi technocratiques et anachroniques soient dénoncées par les sociologues, avant d'être fustigées, au même titre que les grands ensembles mais de manière beaucoup plus marginale, par la critique de l'urbanisme fonctionnaliste. 


\section{BIBLIOGRAPHIE}

Arsène-Henry X., 1962, « Une conception de ville nouvelle », Le groupe familial. Revue publiée par l'École des parents et des éducateurs, $\mathrm{n}^{\text {os }}$ 15-16, avril-juillet, p. 18-23.

Bardet G., 1948, « Les échelons communautaires dans les agglomérations urbaines », in Bardet G., Pierre sur pierre, Paris, LCB éditions, p. 233-249.

Bardet G., 1950, «L'organisation polyphonique appliquée à la composition des grands ensembles ", L'Architecture française, $\mathrm{n}^{\circ}$ 101-102, p. 3-15.

Bardet G., 1957, « Caractère organique des tissus urbains », Urbanisme, 25e année, $\mathrm{n}^{\circ}$ 54, p. 85-92. Bonifazio P., Scrivano P., 2001, Olivetti costruisce : architettura moderna a Ivrea, Milan, Skira. Burgess E. W., 1925, « Can Neighborhood Work have a Scientific Basis? », in Park R. E., Burgess E. W., McKenzie R. D., 1967, The City, Chicago University of Chicago Press, p. 142-155.

Calandra R., 1946, « La teoria americana della "Neighborhood Unit” ", Metron, nº 6, p. 58-59.

Carpenter N., 1933, Encyclopediae of the Social Sciences, New York, The Macmillan Company, vol. XI. Chombart de Lauwe P.-H., 1963, Des Hommes et des villes, Paris, Petite Bibliothèque Payot. Coustenoble A., 1959, « Garges-lès-Gonesse. La Dame Blanche », Urbanisme, $28^{\mathrm{e}}$ année, nº 62-63, p. 102-103.

Dahir J., 1950, Communities for better Living. Citizen Achievement in Organization, Design and Development, New York, Harper and Brothers.

Dal Co F., 1973, « Dai parchi alla regione. L'ideologia progressista e la riforma della città americana », in Ciucci G., Dal Co F., Manieri-Elia M., Tafuri M., La città americana dalla guerra civile al New Deal, Rome-Bari, Laterza, p. 149-314.

Devillard V., Jannière H., 1997a, Espaces publics, communauté et voisinage dans la Reconstruction allemande (1945-1965), Ministère de l'Équipement, Plan Construction Architecture, 129 p.

Devillard V., Jannière H., 1997b, « Espaces publics, communauté et voisinage dans la Reconstruction allemande (1945-1965) », in Picon Lefèvre V. (dir.), 1997, Les espaces publics modernes, Paris, Le Moniteur, p. 15-32.

Di Biagi P., 2001, La grande ricostruzione. Il piano INA-Casa e l'Italia degli anni cinquanta, Rome, Donzelli.

Dieudonné P., 1996, « Et pendant ce temps-là... les autres trente glorieuses ", in Bland-Coquand C., Heudron C., Le Gad R., À la recherche de la ville perdue, actes du colloque des 13 et 14 octobre 1994, Brest, Institut de Géoarchitecture, Paris, L’Harmattan, p. 40-53.

Dupont G., 1959, « Le grand ensemble, facteur de progrès social et de progrès humain ", Urbanisme, $\mathrm{n}^{\circ}$ 62-63, 1959.

Durth W., Gutschow N., 1988, Traüme in Trümmern. Planungen zum Wiederaufbau zerstörter Städte 1940-1950, Mayence, Wiesbaden, Vieweg Verlag.

Fourcaut A., 2004, « Les premiers grands ensembles en région parisienne. Ne pas refaire la banlieue? », French Historical Studies, vol. 27, n 1, winter, p. 195-218. 
Gérard A., 1977, «Quartier et unité de voisinage dans la pratique urbanistique française 1919-1973 », thèse de $3^{\text {e }}$ cycle en géographie, Université de Paris VII, 395 p. (rééd. Unité pédagogique d'architecture de Strasbourg, 1980, 412 p.).

Hamm B., 1973, Betrifft : Nachbarschaft. Verständigung über Inhalt und Gebrauch eines vieldeutiges Begriffs (Voisinage. Le consensus sur le contenu et l'usage d'une notion polysémique), Düsseldorf, Bertelsmann Fachverlag.

Johnson D. L., 2002, « Origin of the Neighbourhood Unit », Planning Perspectives, $\mathrm{n}^{\circ}$ 17, juillet, p. 227-245.

Klages H., 1968, Der Nachbarschaftsgedanke und die nachbarliche Wirklichkeit in der Großstadt (La pensée du voisinage et la réalité du voisinage dans la grande ville), Stuttgart, Kölhammer (1 ${ }^{\text {ère }}$ éd. en 1958).

Lefebvre H., 1960, « Les nouveaux ensembles urbains. Un cas concret : Lacq-Mourenx et les problèmes urbains de la nouvelle classe ouvrière », Revue française de sociologie, vol. I, $\mathrm{n}^{\circ} 2$, avriljuin, p. 186-201.

Mann P. H., 1965, An Approach to Urban Sociology, Londres, New York, p. 155-160.

Mumford L., 1968, « Neighborhood and the Neighborhood Unit », Town Planning Review, janvier 1954, repris in The Urban Prospect, New York, Harcourt, Brace and World, p. 56-78.

Parfait F., 1959, «Conception, organisation, réalisation des ensembles d'habitation », Urbanisme, $28^{\mathrm{e}}$ année, $\mathrm{n}^{\circ} 65$, p. 18-39.

Perry C. A., 1926, « The Local Community as a Unit in the Planning of Urban Residential Areas », in American Sociological Society, The Urban Community, Chicago, University of Chicago Press, p. 238-241.

Perry C. A., 1929, « The Neighborhood Unit », Regional Survey of New York and its Environs, New York, vol. VII.

Pfeil E., 1972, Großstadtforschung, Hanovre, Gebr. Jänecke Verlag ( $2^{\mathrm{e}}$ édition augmentée, $1^{\text {ère }}$ éd. 1948).

Reichow H.-B., 1948, Organische Stadtbaukunst, Brunswick, Georg Werstermann.

Suquet-Bonneaud A., 1950, « L'aménagement des quartiers de résidence en Angleterre », Urbanisme, $\mathrm{n}^{\text {os }} 1-2$, p. 35-48.

Tafuri M., 1990, History of Italian Architecture, 1944-1985, Cambridge, Mass., MIT Press.

Tönnies F., 1887, Gemeinschaft und Geselleschaft. Grundbegriffe der reinen Soziologie ; traduction française, Communauté et société : catégories fondamentales de la sociologie pure, Paris, Retz-CEPL, 1977.

\section{NOTES}

1. Voir par exemple les cycles décrits par Le Corbusier, en relation avec la course du soleil (Manière de penser l'urbanisme).

2. Pour la réception européenne, je renvoie aux travaux de $\mathrm{P}$. Bonifazio sur l'unité de voisinage en Italie (Bonifazio, Scrivano, 2001) ; pour la France, à la thèse de $3^{\mathrm{e}}$ cycle déjà ancienne d'A. Gérard sur la France (Gérard, 1977).

3. Les limites de cet article excluent une lecture systématique de la notion de voisinage dans la sociologie urbaine française de l'après-guerre. Nous nous sommes limités à quelques 
observations de ces sociologues sur l'unité de voisinage parues dans des revues destinées aux professionnels de l'aménagement : cette exploration partielle à ce stade serait à poursuivre.

4. Ce rappel s'appuie sur un ensemble d'écrits de l'École de Chicago, et sur les commentaires émis sur l'unité de voisinage dans les années 1950 et 1960 par la sociologie urbaine.

5. C. H. Cooley, Social Organization. A Study of the larger Mind, New York, C. Scribner's sons, cité par

B. Hamm (1973, p. 11-12).

6. Voir le commentaire de P. H. Mann sur la définition de N. Carpenter (Mann, 1965, p. 152-154).

7. Les conceptions du voisinage de l'École de Chicago, selon l'interprétation de L. Mumford, ne sont pas antithétiques à la métropole.

8. R. E. Park, Modèle spatial et ordre moral, 1926, définit le voisinage sur trois niveaux : la distance physique ; le plan institutionnel ; l'intérêt local ; il prend en compte les déplacements physiques, par exemple de la famille à l'école.

9. C. A. Perry diplômé de Cornell University en 1899 a été employé par la Russell Sage Foundation de 1903 à 1937.

10. Pour les mouvements réformateurs américains, voir F. Dal Co, 1973.

11. Les différentes propositions sur le nombre d'habitants et/ou d'élèves sont reportées par J. Dahir (1950, p. 217-220).

12. Ces villes ont connu une importante fortune critique notamment en Italie, ce qui a contribué à la diffusion de la théorie de l'unité de voisinage après 1945.

13. La loi-cadre du 7 août 1957 institue les zones à urbaniser en priorité ou ZUP, confirmées par le décret du 31 décembre 1958.

14. L'introduction de L. Mumford à la seconde édition de l'ouvrage d'E. Howard, Garden-Cities of Tomorrow est traduite dans le premier numéro de Metron en 1945 ; The Culture of Cities (1938) est traduit en italien en 1954.

15. Pour une analyse de la portée idéologique de ces quartiers voir M. Tafuri, 1990. Sur l'histoire de leur édification voir P. Di Biagi, 2001.

16. Pour un bref panorama européen ainsi que pour un exposé plus détaillé concernant l'Allemagne, voir V. Devillard, H. Jannière, 1997a, $1997 b$.

17. H.-B. Reichow reprend des schémas d'organisation conçus dès le début des années 1940 . Voir W. Durth, N. Gutschow, 1988, p. 300.

18. P. Bourdeix, R. Gagès, F. Grimal, 1952-1960, Secteur industrialisé.

19. Vernouillet, 813 logements. Architecte : Gustave Stoskopf. Maître d'ouvrage : SCIC.

20. C'est ainsi que la dénonce la géographe A. Gérard, Quartier et unité de voisinage, op. cit. Dans d'autres cas, comme Clamart - La Plaine de R. Auzelle, ou le SHAPE - village (1950-1952) construit par J. Dubuisson et F. Dumail, ensemble résidentiel près de Saint-Germain-en-Laye, destiné aux familles des officiers d'État-major des armées américaines, cette appellation se justifierait par l'idée de « cohésion » qu'on a voulu conférer à ces quartiers.

21. Présentation de la rédaction pour l'article de C. Le Cœur, «Esquisse pour une unité de voisinage à Cronenbourg ", Urbanisme, $\mathrm{n}^{\text {os }} 1-2,1950$, p. 29.

22. Ibidem, p. 29.

23. «[...] then becomes a focussing point, both physically and psychically, for the life of the neighborhood» (Perry, 1929).

24. P. Sudreau est alors préfet et Commissaire à la Construction et à l'Urbanisme pour la région parisienne. Les travaux de cette Commission se poursuivent jusqu'à l'été 1958 , alors que P. Sudreau a été nommé ministre de la Construction par le général de Gaulle. Voir A. Fourcaut, 2004, p. 201.

25. «La vie dans les grands ensembles », Urbanisme, $31^{\mathrm{e}}$ année, $\mathrm{n}^{\mathrm{os}} 75-76,1962$, p. 26.

26. H. Klages (1958) et B. Hamm (1968) remarquent tous deux que si la langue anglaise permet de distinguer les deux notions, les urbanistes et architectes allemands les ont au contraire souvent associées (Nachbarschaft). Seule E. Pfeil (Großstadtforschung, Hanovre, Gebr. Jänecke Verlag, 1972, 
1re éd. en 1948) distingue Nachbarschaft (sens spatial) de Nachbarlichkeit (sens d'ensemble de relations sociales).

27. A. Coustenoble, «Le groupement élémentaire », Urbanisme, $28^{\mathrm{e}}$ année, $\mathrm{n}^{\circ}$ 62-63, 1959, p. 103.

\section{RÉSUMÉS}

Cet article examine la diffusion de l'unité de voisinage en France après 1945, sa signification dans les discours urbanistiques, dans la conception puis la critique des grands ensembles. Élaborée au début des années 1920 aux États-Unis par Clarence Arthur Perry à partir des définitions sociologiques du voisinage de l'École de Chicago, l'unité de voisinage a été conçue en rapport avec les déplacements quotidiens, avec la fréquentation quotidienne de l'école primaire ou du centre civique. Connue en France dès 1945 par des articles sur les villes nouvelles britanniques, l'unité de voisinage n'est appliquée qu'à partir de 1951 à quelques projets de quartiers. Sa généralisation, plus tardive qu'en Grande Bretagne ou en Italie, advient surtout lors de la promulgation du décret sur les ZUP : elle est ainsi associée à la grille des équipements (1958). Elle est alors concomitante de la réflexion sur les "échelons » des ensembles d'habitation et les équipements associés. Parallèlement, l'unité de voisinage vue par les architectes, est mise en relation avec la composition et la plastique architecturale. La notion de voisinage invoquée par les architectes et les urbanistes dans les nouveaux quartiers - via l'instrument unité de voisinage - a parfois été appliquée comme si, de fait, elle avait le pouvoir d'instaurer des relations de sociabilité ou de promouvoir des qualités de «vie en commun ». L'article aborde enfin quelques aspects de la critique de cette notion à la fin des années 1960 par les sociologues et les observateurs des pratiques sociales et de la vie quotidienne.

This article looks into the spread of the neighourhood unit in France after 1945, its meaning for town planning, conception, and criticism of high-rise buildings. The neighbourhood unit, which was developed in the United States in the early twenties by Clarence Arthur Perry based on the sociological neighborhood concepts of the Chicago school, was conceived in relation to daily travelling and daily attendance of primary schools or community centres. Whilst the neighourhood unit had been known in France since 1945 through the publication of articles on British new towns, it was applied to just a few area projects from 1951 onwards, later than in Great Britain or Italy. In particular, it was implemented when the decree on ZUPs (priority urban development zones) was issued and was associated with the facilities scale (1958). It coincided with the reflection on the different "levels" of housing developments and their associated facilities. At the same time, the neighbourhood unit viewed by architects is analysed with regard to architectural composition and plasticity. The neighbourhood concept invoked by architects and town planners in new areas - through the neighbourhood unit instrument - was sometimes applied as if it could actually set up relationships based on sociability or promote qualities of "community life". Lastly, the article addresses aspects of the criticism of this concept made by sociologists and observers of social practices and daily life in the late sixties. 
INDEX

Mots-clés : unité de voisinage, grands ensembles, urbanisme, quotidien, architecture, voisinage Index géographique : France

\author{
AUTEUR

\section{HÉLĖNE JANNIÈRE} \\ Historienne de l'architecture \\ École d'architecture Paris-Val de Seine, Ladyss (Laboratoire dynamiques sociales et \\ recomposition des espaces), CNRS/Univ. de Paris 1 \\ 2 rue Valette, 75005 Paris \\ helene.janniere@univ-paris1.fr
}

